\title{
Rigidity, Magnetic Field, and Inflector Voltage Based on Frequency Measurements in Booster
}

\author{
C.J. Gardner
}

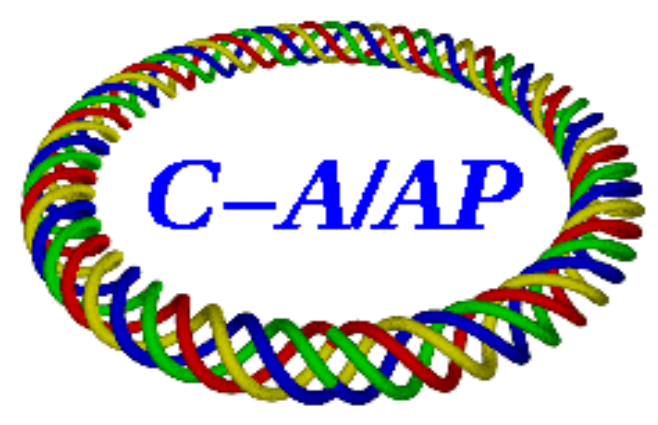

Collider-Accelerator Department

Brookhaven National Laboratory

Upton, NY 11973 


\title{
Rigidity, Magnetic Field, and Inflector Voltage Based on Frequency Measurements in Booster
}

\author{
C.J. Gardner
}

December 5, 2003

Over the course of several months, beginning in October 2002 and ending in July 2003, a number of different ions $\left(\mathrm{D}^{+}, \mathrm{C}^{6+}, \mathrm{Si}^{5+}, \mathrm{Ti}^{18+}, \mathrm{Fe}^{10+}\right.$, $\mathrm{Fe}^{20+}$, and $\mathrm{Au}^{32+}$ ) from Tandem were injected and accelerated in Booster. These were injected at several different magnetic rigidities and accelerated to various energies. For each ion the revolution frequency was measured at injection and top energy. The magnetic field at injection was measured with the Booster Hall probe and Gauss clock. The field in TTB transport line dipole 11DH1 was recorded for each ion. (This dipole is the first of two $90^{\circ}$ bends in the TTB line. It is used to select the momentum of ions to be transported to Booster. Its field is monitored by an NMR probe.) The C3 electrostatic inflector setpoint was recorded as was the BMM (Booster Main Magnet) field setpoint at extraction.

Assuming a given equilibrium orbit radius, the frequency measurements give the momentum and magnetic rigidity of each ion. (We do not yet have a working orbit acquisition system, so the orbit radius cannot be measured.) These data are used to obtain the relation between the ion momentum (or rigidity) and the measured or set fields. The assumed orbit radius also gives the radius of curvature in the Booster dipoles. This together with the magnetic rigidity gives a value for the field in the dipoles. The ion momentum also gives a value for the voltage required between the cathode and septum of the inflector.

Having the relations between ion momentum and the measured fields is extremely valuable. These allow one to obtain the ion momentum from the measured field in the 11DH1 dipole and then to verify that the measured field in Booster at injection has the correct value for this momentum. This means that one does not have to search for the correct injection field to get beam going around the machine. Similarly the relation between the ion 
momentum and the inflector setpoint allows one to set the inflector voltage to the correct value for a given ion momentum. The relation between the ion momentum and magnetic field setpoint at extraction allows one to set the extraction field to the correct value for the desired momentum.

In the following, we report on the frequency and field measurements and the resulting relations between ion momentum, measured and set fields, and inflector setpoint. The necessary formulae are given in Sections 1 and 2. The measurements and resulting relations are presented in Sections 3 through 6 .

\section{Formulae for Rigidity and Field in Terms of Frequency and Radius}

Consider a particle of mass $m$ moving along an orbit of radius $R$ with velocity $v$. In terms of the radius and the revolution frequency, $f$, the velocity is

$$
v=2 \pi R f .
$$

This gives momentum, energy, and kinetic energy

$$
p=m c \beta \gamma, \quad E=m c^{2} \gamma=\sqrt{c^{2} p^{2}+m^{2} c^{4}}, \quad W=E-m c^{2}
$$

where

$$
\beta=v / c, \quad \gamma=1 / \sqrt{1-\beta^{2}} .
$$

Let $d f$ and $d R$ be the errors in the frequency and radius measurements.

Then the error in $v$ is given by

$$
\frac{d v}{v}=\frac{d f}{f}+\frac{d R}{R}
$$

Differentiating $p$ with respect to $v$ we obtain

$$
\frac{d p}{d v}=m \gamma^{3}=\frac{p}{v} \gamma^{2}
$$

and therefore

$$
\frac{d p}{p}=\gamma^{2} \frac{d v}{v}=\gamma^{2}\left\{\frac{d f}{f}+\frac{d R}{R}\right\}
$$

which gives the error in momentum. Note that the fractional error $d p / p$ is proportional to $\gamma^{2}$ which is large for relativistic particles but is of order one for nonrelativistic particles. 
The magnetic rigidity of the particle in units of $\mathrm{Tm}$ is

$$
B \rho=k p / Q
$$

where $Q$ is the particle charge in units of the proton charge, $p$ is the momentum in units of $\mathrm{GeV} / \mathrm{c}$, and $k=10^{9} / 299792458$. The radius of curvature is

$$
\rho=\rho_{0}\left(R / R_{0}\right)^{1 / \alpha}
$$

where

$$
\rho_{0}=13.8656 \mathrm{~m}, \quad R_{0}=201.78 /(2 \pi) \mathrm{m}
$$

and

$$
1 / \alpha=\gamma_{t}^{2}, \quad \gamma_{t}=4.806
$$

Here $\rho_{0}$ is the nominal radius of curvature in the Booster dipoles, $R_{0}$ is the nominal radius, and $\gamma_{t}$ is the transition gamma. Equation (8) is derived in the Appendix. The errors in $B \rho$ and $\rho$ due to the frequency and radius errors are given by

$$
\frac{d(B \rho)}{B \rho}=\frac{d p}{p}=\gamma^{2}\left\{\frac{d f}{f}+\frac{d R}{R}\right\}
$$

and

$$
\frac{d \rho}{\rho}=\gamma_{t}^{2} \frac{d R}{R}
$$

Having obtained $B \rho$ and $\rho$, the magnetic field is

$$
B=(B \rho) / \rho
$$

and the error in $B$ is given by

$$
\frac{d B}{B}=\frac{d(B \rho)}{B \rho}-\frac{d \rho}{\rho}=\gamma^{2} \frac{d f}{f}+\left(\gamma^{2}-\gamma_{t}^{2}\right) \frac{d R}{R} .
$$

Here we see that for nonrelativistic particles, the contribution of the radial error to $d B / B$ can be much larger than the contribution of the frequency error. 


\section{Formulae for Inflector Voltage}

The voltage $V_{I}$ required for particles with momentum $p$ and charge $Q$ to follow the nominal trajectory through the inflector is given by

$$
e V_{I}=\frac{G}{R_{I}}\left(\frac{c^{2} p^{2}}{Q E}\right)
$$

Here $G=0.017 \mathrm{~m}$ is the gap between the cathode and septum of the inflector, $R_{I}=8.74123 \mathrm{~m}$ is the radius of curvature along the nominal trajectory, and the momentum and energy are given in terms of frequency and radius by equations (1-3). The errors in $p$ and $E$ are given by

$$
\frac{d p}{p}=\gamma^{2}\left\{\frac{d f}{f}+\frac{d R}{R}\right\}, \quad \frac{d E}{E}=\beta^{2} \frac{d p}{p}
$$

which give

$$
\frac{d V_{I}}{V_{I}}=2 \frac{d p}{p}-\frac{d E}{E}=\left(2-\beta^{2}\right) \gamma^{2}\left\{\frac{d f}{f}+\frac{d R}{R}\right\}
$$

for the error in $V_{I}$.

\section{Frequency Measurement}

The revolution frequency was measured by observing the turn-by-turn sum signal from a Booster BPM. This signal is captured on an oscilliscope and analyzed by the Turn-by-Turn Program which displays the trace of each turn above the traces of the previous turns. The frequency in the program is adjusted so that the beam bunches on the traces line up vertically. This is the measured frequency. One can get some sense of the error in the measurement by seeing how much the set frequency has to be shifted to see that the bunches no longer line up vertically.

Since the beam coming into Booster from Tandem is not bunched, the revolution frequency at injection normally cannot be seen on a BPM. To put some structure on the incoming beam so that the revolution frequency can be seen, a half-turn is chopped out of the beam before injection as described in Ref. [1]. (Occasionally there is enough structure on the unchopped beam to see the revolution frequency but we do not depend on this to make measurements.)

Turn-by-turn frequency measurements were made by a number of people (L.A. Ahrens, K.A. Brown, C.J. Gardner, and N. Tsoupas) at various times 
over the course of several months. For most of the measurements a careful estimate of the error was not done. The bunches in the turn-by-turn display were simply lined up vertically as best as could be done by eye.

\section{Rigidity and Field from Frequency Measurements at Injection}

Tables 1, 2, and 3 list the measured frequencies for various ions at Booster injection. These were used to calculate the kinetic energy $W$, magnetic rigidity $B \rho$, field $B$, and inflector voltage $V_{I}$. The orbit radius was assumed to be the nominal radius $R_{0}=201.78 /(2 \pi) \mathrm{m}$. The estimated fractional errors in the frequency and radius are

$$
\frac{d f}{f} \approx 3 \times 10^{-4}, \quad \frac{d R}{R} \approx 3 \times 10^{-4}
$$

which give

$$
\frac{d(B \rho)}{B \rho}=\frac{d p}{p}=\gamma^{2}\left\{\frac{d f}{f}+\frac{d R}{R}\right\} \approx 6 \times 10^{-4}
$$

and

$$
\frac{d B}{B}=\gamma^{2} \frac{d f}{f}+\left(\gamma^{2}-\gamma_{t}^{2}\right) \frac{d R}{R} \approx 7 \times 10^{-3} .
$$

Magnetic field measurements from the 11DH1 NMR probe, Booster Hall probe, and Gauss clock are also listed in the Tables along with the C3 inflector voltage setpoint $V_{S}$. The Hall probe reading is the value of the dwell field prior to injection. The Gauss clock gives the change in field from BT0 to the time of injection. (We assume that the field at BT0 is equal to the dwell field.) The measured injection field $H$ is then defined to be the sum of the Hall probe and Gauss clock readings.

Table 4 summarizes the rigidity and field data of Tables 1, 2, and 3. Here $B_{N}$ is the reading of the NMR probe in TTB dipole $11 \mathrm{DH} 1$.

Figure 1 is a plot of the $B$ and $H$ data from Table 4 with fitted line $B=m H+b$. The fitted parameters are $m=1.00076(125)$ and $b=4.81(79)$ Gauss. The indicated errors were obtained assuming a statistical error of $\sigma=0.56$ Gauss for each value of $B$. This gives a $\chi^{2}$ per dof (degree of freedom) of 1 . Figure 2 shows the deviation of the Figure 1 data points from the fitted line.

Figure 3 is a plot of the $B \rho$ and $B_{N}$ data from Table 4 with fitted line $B \rho=m B_{N}+b$. The fitted parameters are $m=1.53065(98) \times 10^{-4} \mathrm{Tm}$ per 
Gauss and $b=-5.44(5.70) \times 10^{-4} \mathrm{Tm}$. The indicated errors were obtained assuming a statistical error of $\sigma=0.0004 \mathrm{Tm}$ for each value of $B \rho$. This gives a $\chi^{2}$ per dof of 1 . Figure 4 shows the deviation of the Figure 3 data points from the fitted line.

Having obtained the values of parameters $m$ and $b$ in the relations $B \rho=m B_{N}+b$ and $B=m H+b$, we can substitute the measured 11DH1 dipole field $B_{N}$ into the first relation to obtain the magnetic rigidity of any ion being transported to Booster. Assuming the nominal radius of curvature in the Booster dipoles then gives the field $B$ required for the ions to follow the nominal orbit in Booster. This then can be used in the second relation to obtain the desired injection field $H$ to be measured by the Hall probe and Gauss clock. The rigidity also can be used in (7) to obtain the ion momentum $p$. This in turn can be used in (15) to calculate the inflector voltage $V_{I}$.

\section{$5 \quad V_{I}$ from Frequency Measurements at Injection}

Table 5 summarizes the inflector voltage data from Tables 1, 2, and 3. Here $V_{I}$ is voltage determined from the frequency measurements and radius. $V_{S}$ is the voltage setpoint. Using the estimated fractional errors in frequency and radius given by (18), we have

$$
\frac{d V_{I}}{V_{I}}=\left(2-\beta^{2}\right) \gamma^{2}\left\{\frac{d f}{f}+\frac{d R}{R}\right\} \approx 12 \times 10^{-4}
$$

Figure 5 is a plot of the $V_{I}$ and $V_{S}$ data from Table 5 with fitted line $V_{I}=m V_{S}+b$. The fitted parameters are $m=0.9858(44)$ and $b=0.024(199) \mathrm{kV}$. The indicated errors were obtained assuming a statistical error of $\sigma=0.17 \mathrm{kV}$ for each value of $V_{I}$. This gives a $\chi^{2}$ per dof of 1 . Figure 6 shows the deviation of the Figure 5 data points from the fitted line.

Having obtained the values of parameters $m$ and $b$ in the relation $V_{I}=m V_{S}+b$, we can obtain the desired setpoint $V_{S}$ once $V_{I}$ has been determined from the 11DH1 dipole field $B_{N}$ as discussed above. 


\section{Rigidity and Field from Frequency Measurements at Extraction}

Tables 6, 7, and 8 list the measured frequencies for various ions at Booster extraction. These were used to calculate the kinetic energy $W$, magnetic rigidity $B \rho$, and field $B$. The orbit radius was assumed to be the nominal radius $R_{0}=201.78 /(2 \pi) \mathrm{m}$. The setpoint of the field at extraction is also listed. This is the programmed value of the field in the BMM program. The calculated MM current is equal to the calculated field $B$ (in Gauss) divided by 2.43 Gauss per Amp [2]. The MM current setpoint is the programmed value of the current in the BMM program.

Table 9 summarizes the rigidity and field data of Tables 6, 7, and 8. Here $B_{0}$ is the field setpoint at extraction.

Figure 7 is a plot of the $B$ and $B_{0}$ data from Table 9 with fitted line $B=m B_{0}+b$. The fitted parameters are $m=0.98710(81)$ and $b=-24.9(7.4)$ Gauss. The indicated errors were obtained assuming a statistical error of $\sigma=6.7$ Gauss for each value of $B$. This gives a $\chi^{2}$ per dof of 1 . Figure 8 shows the deviation of the Figure 7 data points from the fitted line.

Having obtained the values of parameters $m$ and $b$ in the relation $B=m B_{0}+b$, we can obtain the setpoint $B_{0}$ required for a given ion rigidity at extraction. Here we assume the nominal radius of curvature in the Booster dipoles. The given rigidity $B \rho$ then gives the field $B$ required for the ions to follow the nominal orbit in Booster at extraction.

Substituting $B$ into the relation $B=m B_{0}+b$ then gives the required setpoint $B_{0}$.

\section{Appendix}

The radius, $R$, of an equilibrium orbit in a synchrotron is defined to be the circumference of the orbit divided by $2 \pi$. Let $R_{0}$ be the radius of the design orbit, and let $p_{0}$ be the momentum of a charged particle traveling along this orbit when the field at a reference point in one of the synchrotron dipoles is $b_{0}$. We shall assume that when the field at the reference point is changed to $b$, the field at every other point in the synchrotron aperture is scaled by the same factor $b / b_{0}$. We assume further that the radius of the equilibrium orbit is a unique function of $b$ and the 
particle momentum $p$. Thus we can write

$$
R=R(p, b), \quad R_{0}=R\left(p_{0}, b_{0}\right) .
$$

Now, if we change the momentum from $p$ to $p+h$ and scale the field $b$ by the same factor $(p+h) / p$, then the particle will follow the same orbit it did when the momentum and field were $p$ and $b$. The function $R(p, b)$ therefore has the property

$$
R(p+h, b+b h / p)=R(p, b)
$$

and it follows that

$$
\frac{\partial R}{\partial p}+\frac{b}{p} \frac{\partial R}{\partial b}=0
$$

Thus

$$
d R=\frac{\partial R}{\partial p} d p+\frac{\partial R}{\partial b} d b=p \frac{\partial R}{\partial p}\left(\frac{d p}{p}-\frac{d b}{b}\right)
$$

and defining momentum compaction factor

$$
\alpha=\frac{p}{R} \frac{\partial R}{\partial p}=-\frac{b}{R} \frac{\partial R}{\partial b}
$$

we have

$$
\frac{d R}{R}=\alpha\left(\frac{d p}{p}-\frac{d b}{b}\right)=\frac{1}{\gamma_{t}^{2}}\left(\frac{d p}{p}-\frac{d b}{b}\right)
$$

where

$$
\gamma_{t}^{2}=1 / \alpha
$$

For sufficiently small regions of the $p, b$ space we shall assume that $\alpha$ is constant. Then writing the first of equations (26) as

$$
\frac{1}{R} \frac{\partial R}{\partial p}=\frac{\alpha}{p}
$$

and integrating from $p_{0}$ to $p$ we have

$$
\ln R(p, b)-\ln R\left(p_{0}, b\right)=\ln p^{\alpha}-\ln p_{0}^{\alpha}
$$

and therefore

$$
R(p, b)=R\left(p_{0}, b\right)\left(p / p_{0}\right)^{\alpha} .
$$

Similarly, writing the second of equations (26) as

$$
\frac{1}{R} \frac{\partial R}{\partial b}=-\frac{\alpha}{b}
$$


and integrating from $b_{0}$ to $b$ we have

$$
\ln R\left(p_{0}, b\right)-\ln R\left(p_{0}, b_{0}\right)=-\ln b^{\alpha}+\ln b_{0}^{\alpha}
$$

and therefore

$$
R\left(p_{0}, b\right)=R\left(p_{0}, b_{0}\right)\left(b_{0} / b\right)^{\alpha} .
$$

Finally, putting (34) into (31) we have

$$
R(p, b)=R_{0}\left(\frac{b_{0}}{b} \frac{p}{p_{0}}\right)^{\alpha} .
$$

This is the fundamental relation between field, momentum, and radius. In terms of the radii of curvature

$$
\rho_{0}=\frac{c p_{0}}{e b_{0}}, \quad \rho=\frac{c p}{e b}
$$

we then have

$$
R=R_{0}\left(\rho / \rho_{0}\right)^{\alpha}, \quad \rho=\rho_{0}\left(R / R_{0}\right)^{1 / \alpha} .
$$

\section{References}

[1] C.J. Gardner, L.A. Ahrens, and N. Williams, "Turn-by-Turn Analysis of Proton and Gold Beams at Injection in the AGS Booster", Proceedings of the 1999 Particle Accelerator Conference, New York, 2063-2065 (1999)

[2] R. Thern, "Booster Dipole Production Measurements", Booster Tech. Note 190, March 13, 1991. 
Table 1: Gold and Deuteron Parameters at Booster Injection

\begin{tabular}{|c|c|c|c|c|}
\hline Parameter & $\mathrm{Au}^{32+}$ & Deuteron & Deuteron & Unit \\
\hline$m c^{2}$ & 183.456812 & 1.875612762 & 1.875612762 & $\mathrm{GeV}$ \\
\hline Date & 10 Oct 02 & 12 Oct 02 & 21 Nov 02 & \\
\hline $\begin{array}{c}11 \mathrm{DH} 1 \\
\text { NMR Probe }\end{array}$ & 5580 & 4640 & 5580 & Gauss \\
\hline$h f$ & $397.74(06)$ & $502.60(15)$ & 401.922 & $\mathrm{kHz}$ \\
\hline$h$ & 6 & 3 & 2 & \\
\hline$T=1 / f$ & 15.0852 & 5.9690 & 4.9761 & $\mu \mathrm{s}$ \\
\hline $\begin{array}{c}\text { Kinetic } \\
\text { Energy } W\end{array}$ & 182.8790 & 12.0392 & 17.3965 & $\mathrm{MeV}$ \\
\hline$B \rho$ & 0.854085 & 0.710002 & 0.854085 & $\mathrm{Tm}$ \\
\hline$B \rho / \rho$ & 615.974 & 512.060 & 615.974 & Gauss \\
\hline $\begin{array}{c}\text { Booster } \\
\text { Hall Probe }\end{array}$ & 593.7 & 492.6 & 593.7 & Gauss \\
\hline $\begin{array}{c}\text { Booster } \\
\text { Gauss Clock }\end{array}$ & 16.7 & 13.3 & 16.7 & Gauss \\
\hline $\begin{array}{l}\text { Injection } \\
\text { Field } H\end{array}$ & 610.4 & 505.9 & 610.4 & Gauss \\
\hline $\begin{array}{c}\text { Inflector } \\
\text { Setpoint } V_{S}\end{array}$ & 22.538 & 47.396 & 68.070 & $\mathrm{kV}$ \\
\hline $\begin{array}{l}\text { Calculated } \\
\text { Voltage } V_{I}\end{array}$ & 22.218 & 46.678 & 67.355 & $\mathrm{kV}$ \\
\hline
\end{tabular}


Table 2: Iron and Silicon Parameters at Booster Injection

\begin{tabular}{|c|c|c|c|}
\hline Parameter & $\mathrm{Fe}^{10+}$ & $\mathrm{Si}^{5+}$ & Unit \\
\hline$m c^{2}$ & 52.097954 & 26.057785 & $\mathrm{GeV}$ \\
\hline Date & 5 Nov 02 & 15 Nov 02 & \\
\hline $\begin{array}{c}11 \mathrm{DH} 1 \\
\text { NMR Probe }\end{array}$ & 7958.15 & 7958 & Gauss \\
\hline$h f$ & $622.98(12)$ & $622.80(12)$ & $\mathrm{kHz}$ \\
\hline$h$ & 6 & 6 & \\
\hline$T=1 / f$ & 9.6311 & 9.6339 & $\mu \mathrm{s}$ \\
\hline $\begin{array}{c}\text { Kinetic } \\
\text { Energy } W\end{array}$ & 127.6867 & 63.8279 & $\mathrm{MeV}$ \\
\hline$B \rho$ & 1.217428 & 1.217486 & $\mathrm{Tm}$ \\
\hline$B \rho / \rho$ & 878.021 & 878.062 & Gauss \\
\hline $\begin{array}{c}\text { Booster } \\
\text { Hall Probe }\end{array}$ & 824.4 & 820.1 & Gauss \\
\hline $\begin{array}{c}\text { Booster } \\
\text { Gauss Clock }\end{array}$ & 48.0 & 52.8 & Gauss \\
\hline $\begin{array}{c}\text { Injection } \\
\text { Field } H\end{array}$ & 872.4 & 872.9 & Gauss \\
\hline $\begin{array}{c}\text { Inflector } \\
\text { Setpoint } V_{S}\end{array}$ & 50.507 & 50.455 & $\mathrm{kV}$ \\
\hline $\begin{array}{l}\text { Calculated } \\
\text { Voltage } V_{I}\end{array}$ & 49.604 & 49.588 & $\mathrm{kV}$ \\
\hline
\end{tabular}


Table 3: Iron, Titanium, and Carbon Parameters at Booster Injection

\begin{tabular}{|c|c|c|c|c|}
\hline Parameter & $\mathrm{Ti}^{18+}$ & $\mathrm{Fe}^{20+}$ & $\mathrm{C}^{6+}$ & Unit \\
\hline$m c^{2}$ & 44.6540277 & 52.0928437 & 11.1748622 & $\mathrm{GeV}$ \\
\hline Date & 24 June 03 & 2 June 03 & 27 May 03 & \\
\hline $\begin{array}{c}11 \mathrm{DH} 1 \\
\text { NMR Probe }\end{array}$ & 4364.1 & 4364.1 & 4364.1 & Gauss \\
\hline$h f$ & $358.45(10)$ & $341.15(10)$ & $475.89(10)$ & $\mathrm{kHz}$ \\
\hline$h$ & 3 & 3 & 3 & \\
\hline$T=1 / f$ & 8.3694 & 8.7938 & 6.3040 & $\mu \mathrm{s}$ \\
\hline $\begin{array}{c}\text { Kinetic } \\
\text { Energy } W\end{array}$ & 145.101856 & 153.258446 & 64.24366 & $\mathrm{MeV}$ \\
\hline$B \rho$ & 0.667638100 & 0.666936878 & 0.66711783 & $\mathrm{Tm}$ \\
\hline$B \rho / \rho$ & 481.507 & 481.001 & 481.132 & Gauss \\
\hline $\begin{array}{c}\text { Booster } \\
\text { Hall Probe }\end{array}$ & 451.2 & 451.2 & 451.2 & Gauss \\
\hline $\begin{array}{c}\text { Booster } \\
\text { Gauss Clock }\end{array}$ & 25.2 & 25.2 & 25.2 & Gauss \\
\hline $\begin{array}{c}\text { Injection } \\
\text { Field } H\end{array}$ & 476.4 & 476.4 & 476.4 & Gauss \\
\hline $\begin{array}{c}\text { Inflector } \\
\text { Setpoint } V_{S}\end{array}$ & 31.556 & 30.079 & 42.122 & $\mathrm{kV}$ \\
\hline $\begin{array}{l}\text { Calculated } \\
\text { Voltage } V_{I}\end{array}$ & 31.304 & 29.762 & 41.528 & $\mathrm{kV}$ \\
\hline
\end{tabular}


Table 4: Summary of Rigidities and Fields. Units are Tm and Gauss. $B \rho$ and $B$ are determined from frequency measurements and radius. $H$ is the injection field obtained from the Booster Hall probe and Gauss clock. $B_{N}$ is the reading of the NMR probe in TTB dipole $11 \mathrm{DH} 1$.

\begin{tabular}{|c|c|c|c|c|}
\hline Date & $B \rho$ & $B$ & $H$ & $B_{N}$ \\
\hline 10 Oct 02 & 0.854085 & 615.974 & 610.4 & 5580 \\
\hline 12 Oct 02 & 0.710002 & 512.060 & 505.9 & 4640 \\
\hline 5 Nov 02 & 1.217428 & 878.021 & 872.4 & 7958.15 \\
\hline 15 Nov 02 & 1.217486 & 878.062 & 872.9 & 7958 \\
\hline 27 May 03 & 0.667118 & 481.132 & 476.4 & 4364.1 \\
\hline 2 Jun 03 & 0.666937 & 481.001 & 476.4 & 4364.1 \\
\hline 24 Jun 03 & 0.667638 & 481.507 & 476.4 & 4364.1 \\
\hline
\end{tabular}




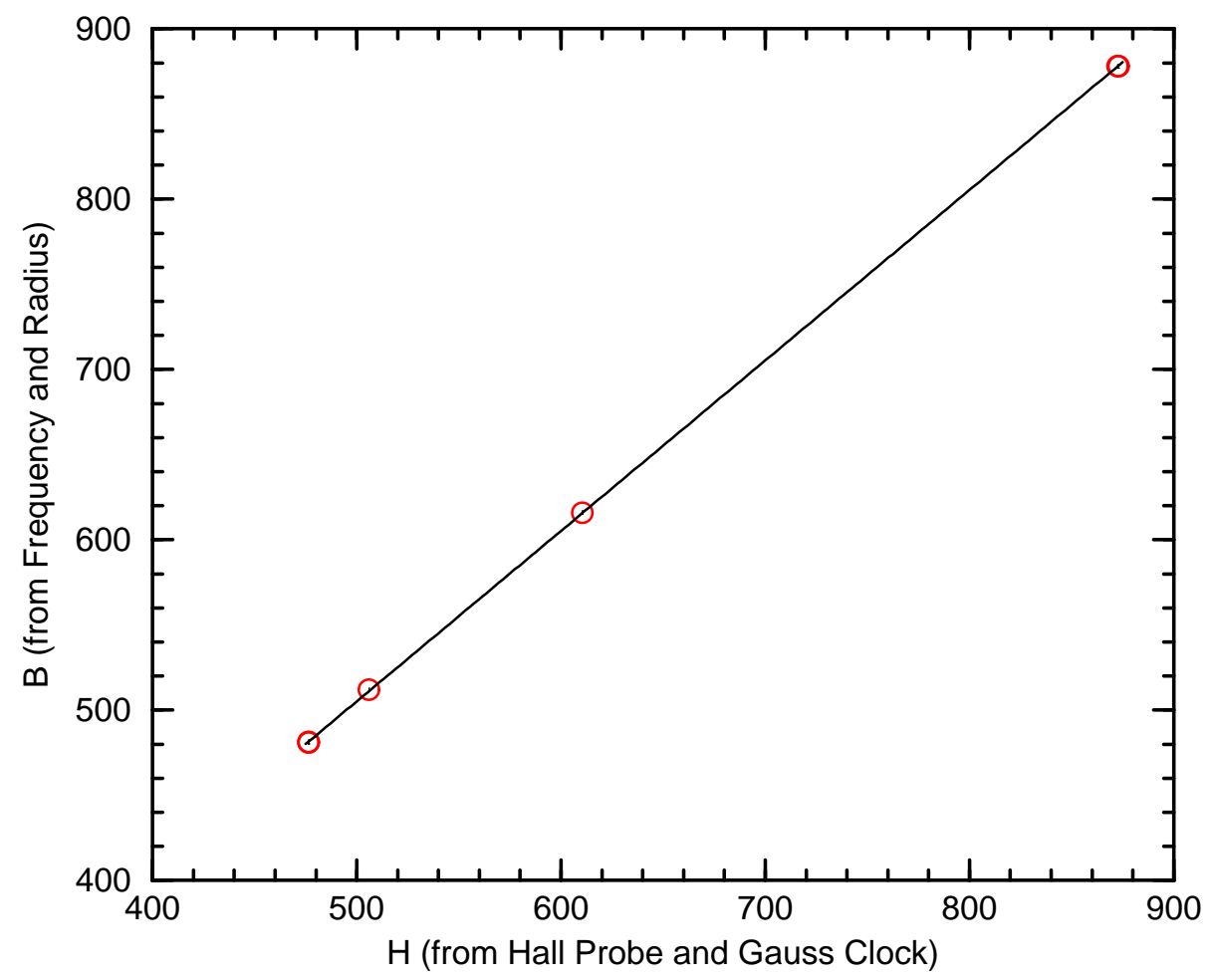

Figure 1: Plot of $B$ and $H$ data from Table 4 with fitted line. Units are Gauss. $B$ is the field determined from frequency and radius. $H$ is the injection field determined from the Booster Hall probe and Gauss clock. The line $B=m H+b$ is fitted to the data points. This gives fitted parameters $m=1.00076(125)$ and $b=4.81(79)$ Gauss. The indicated errors were obtained assuming a statistical error of $\sigma=0.56$ Gauss for each value of $B$. This gives a $\chi^{2}$ per dof of 1 . 


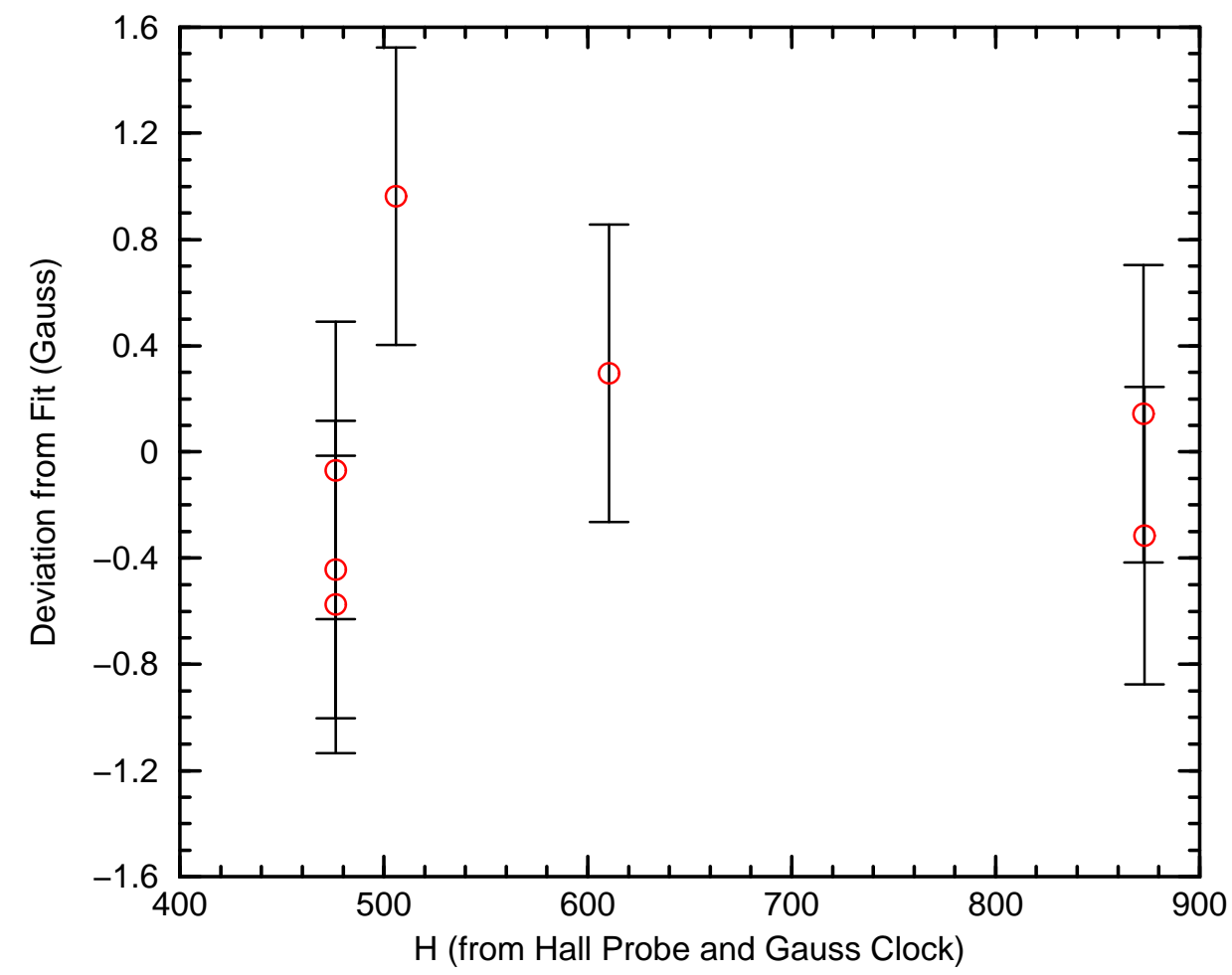

Figure 2: Deviation of Figure 1 data points from fitted line. Units are Gauss. The indicated errors are \pm 0.56 Gauss. 


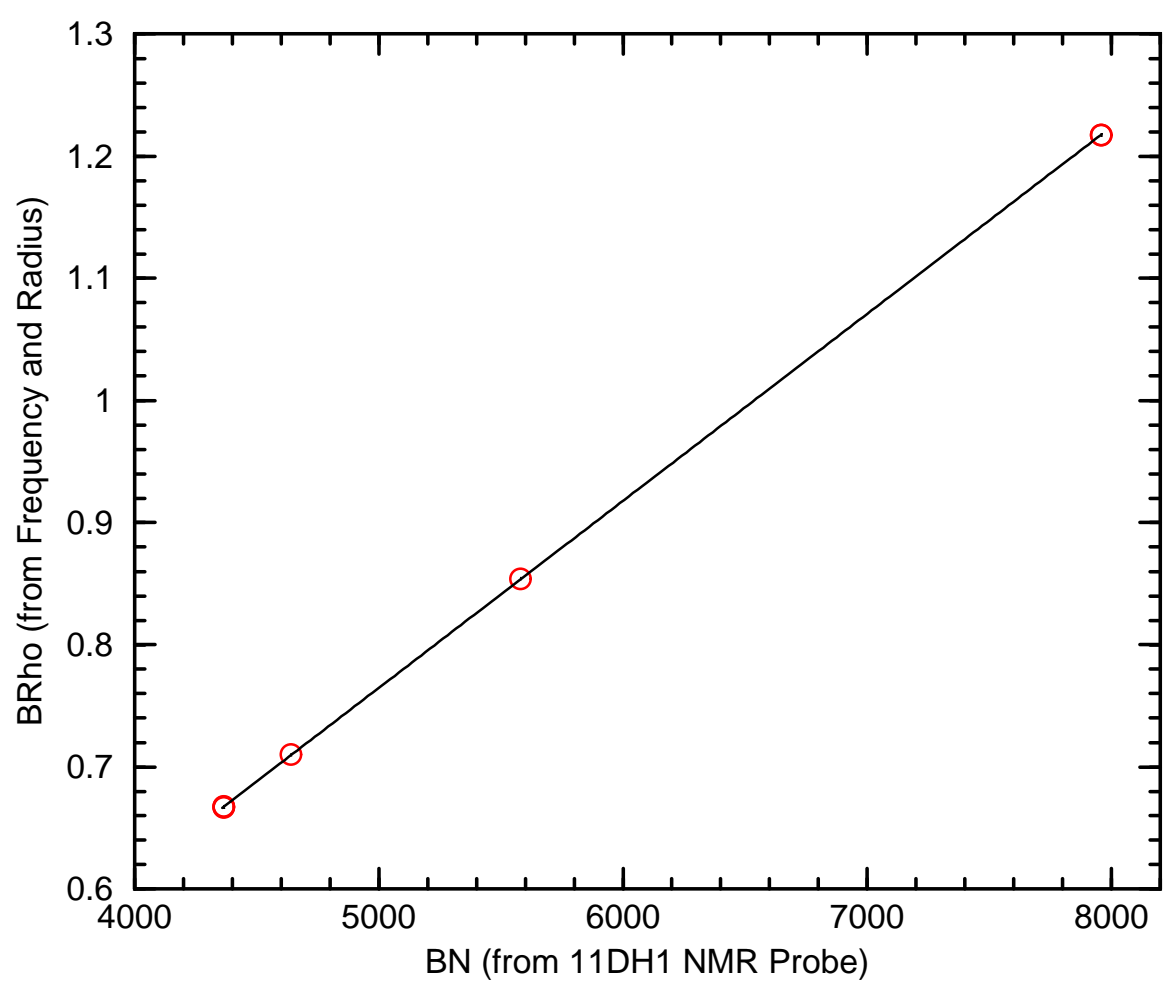

Figure 3: Plot of $B \rho$ and $B_{N}$ data from Table 4 with fitted line. Units are Tm and Gauss. $B \rho$ is the rigidity determined from frequency and radius. $B_{N}$ is the NMR probe reading in TTB dipole 11DH1. The line $B \rho=m B_{N}+b$ is fitted to the data points. This gives fitted parameters $m=1.53065(98) \times$ $10^{-4}$ Tm per Gauss and $b=-5.44(5.70) \times 10^{-4} \mathrm{Tm}$. The indicated errors were obtained assuming a statistical error of $\sigma=0.0004 \mathrm{Tm}$ for each value of $B \rho$. This gives a $\chi^{2}$ per dof of 1 . 


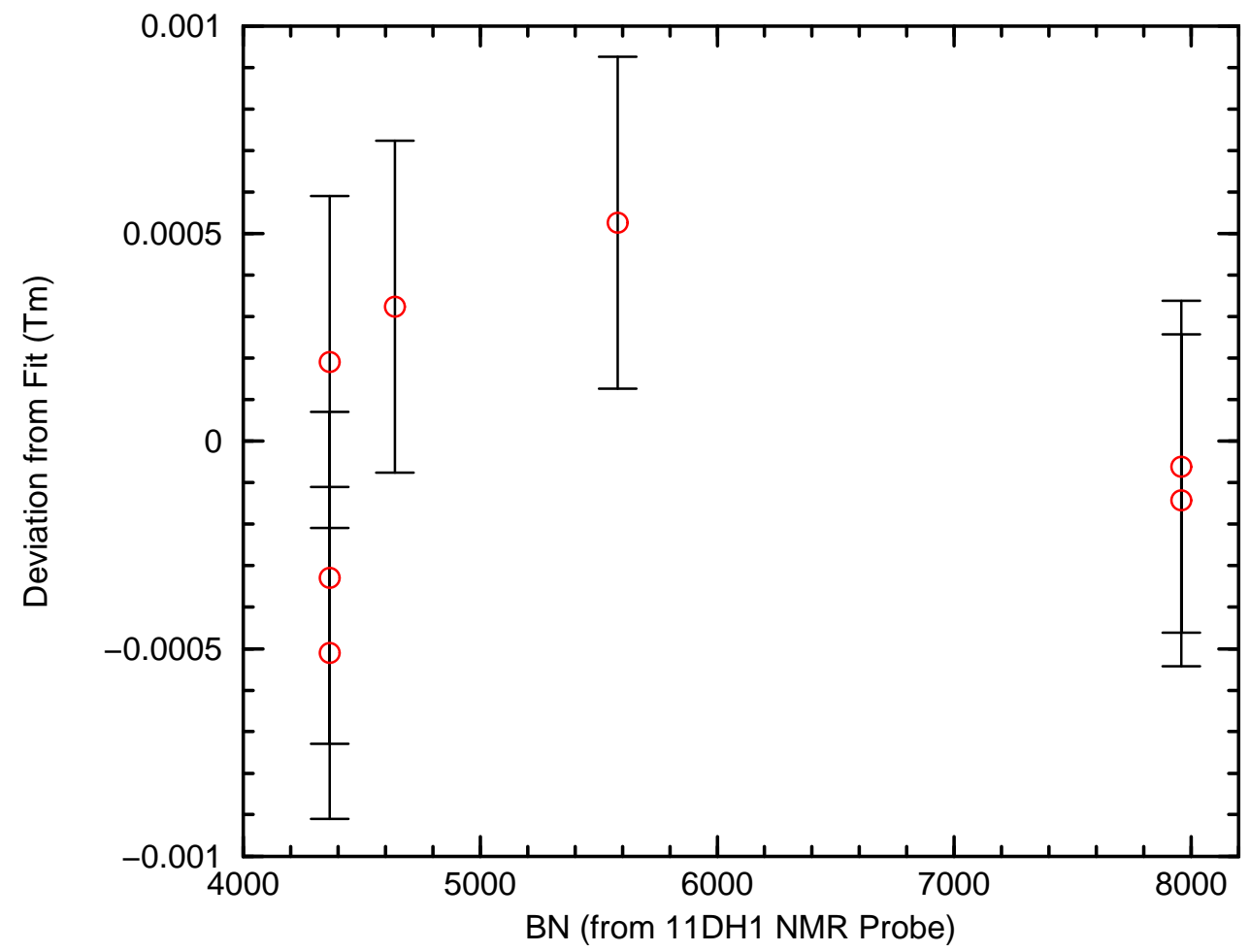

Figure 4: Deviation of Figure 3 data points from fitted line. Units are Tm and Gauss. The indicated errors are $\pm 0.0004 \mathrm{Tm}$. 
Table 5: Summary of Inflector Voltages. Units are kilovolts. $V_{I}$ is determined from frequency measurements and radius. $V_{S}$ is the inflector voltage setpoint.

\begin{tabular}{|c|c|c|}
\hline Date & $V_{I}$ & $V_{S}$ \\
\hline 10 Oct 02 & 22.218 & 22.538 \\
\hline 12 Oct 02 & 46.678 & 47.396 \\
\hline 5 Nov 02 & 49.604 & 50.507 \\
\hline 15 Nov 02 & 49.588 & 50.455 \\
\hline 21 Nov 02 & 67.355 & 68.070 \\
\hline 27 May 03 & 41.528 & 42.122 \\
\hline 2 Jun 03 & 29.762 & 30.079 \\
\hline 24 Jun 03 & 31.304 & 31.556 \\
\hline
\end{tabular}




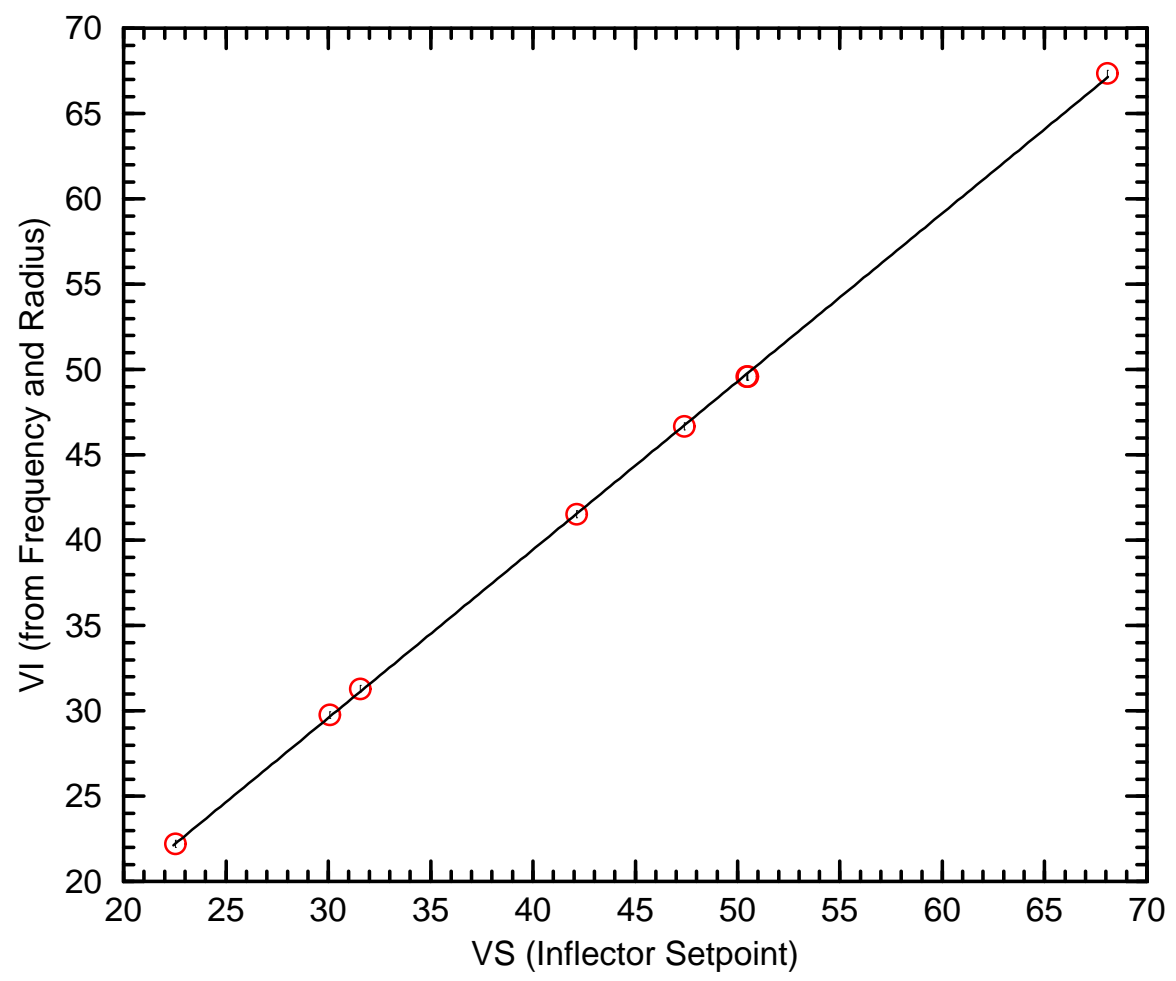

Figure 5: Plot of $V_{I}$ and $V_{S}$ data from Table 5 with fitted line. Units are kilovolts. $V_{I}$ is the inflector voltage determined from frequency and radius. $V_{S}$ is the inflector voltage setpoint. The line $V_{I}=m V_{S}+b$ is fitted to the data points. This gives fitted parameters $m=0.9858(44)$ and $b=0.024(199) \mathrm{kV}$. The indicated errors were obtained assuming a statistical error of $\sigma=0.17$ $\mathrm{kV}$ for each value of $V_{I}$. This gives a $\chi^{2}$ per dof of 1 . 


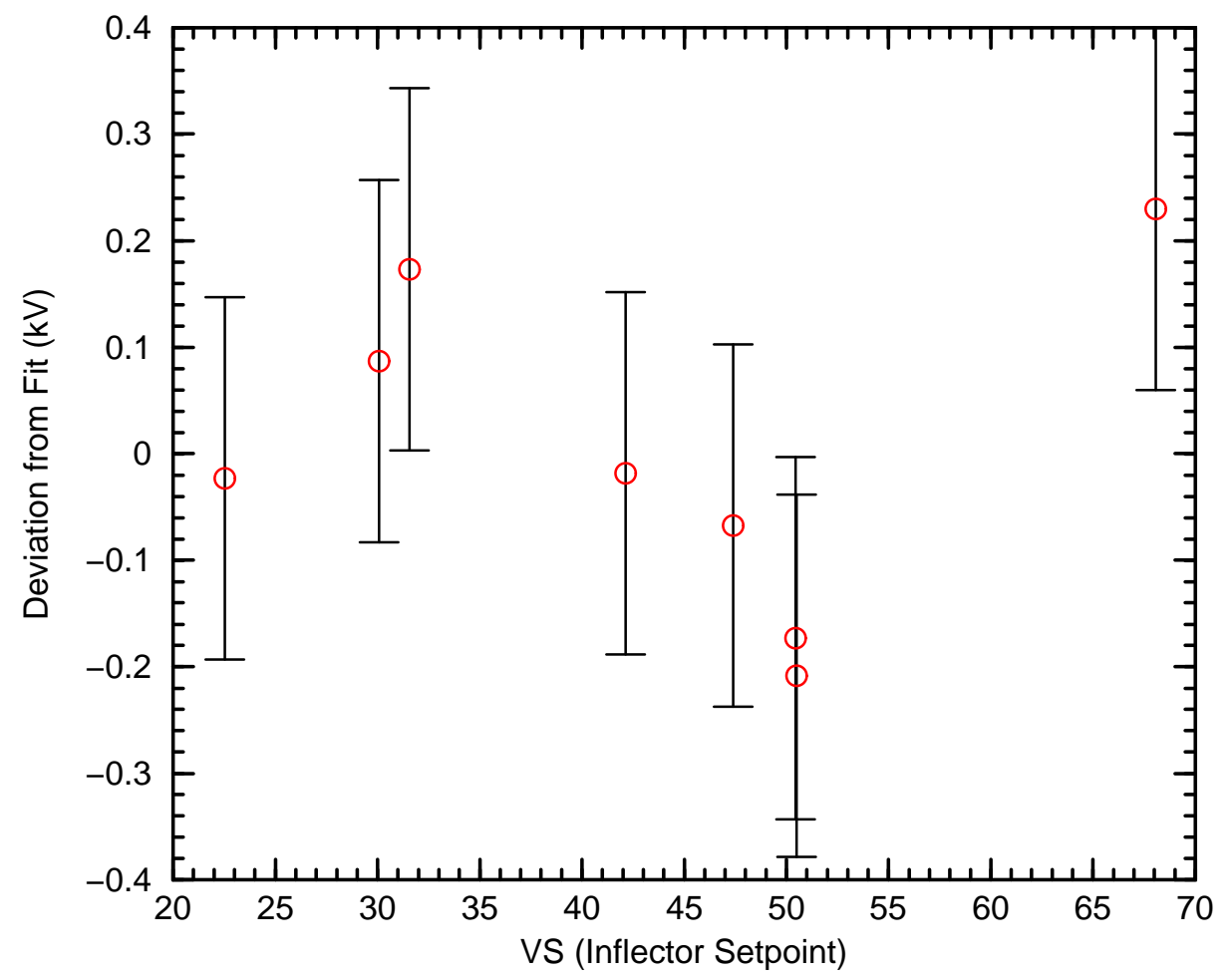

Figure 6: Deviation of Figure 5 data points from fitted line. Units are $\mathrm{kV}$. The indicated errors are $\pm 0.17 \mathrm{kV}$. 
Table 6: Carbon and Iron Parameters at Extraction

\begin{tabular}{|c|c|c|c|c|}
\hline Parameter & $\mathrm{C}^{6+}$ & $\mathrm{Fe}^{20+}$ & $\mathrm{C}^{6+}$ & Unit \\
\hline$m c^{2}$ & 11.1748622 & 52.0928437 & 11.1748622 & $\mathrm{GeV}$ \\
\hline Date & 27 May 03 & 30 May 03 & 9 June 03 & \\
\hline$h f$ & $2.8503(6)$ & $3.68724(60)$ & $2.9208(6)$ & $\mathrm{MHz}$ \\
\hline$h$ & 3 & 3 & 3 & \\
\hline$T=1 / f$ & 1.05252 & 0.81362 & 1.02712 & $\mu \mathrm{s}$ \\
\hline $\begin{array}{c}\text { Kinetic E } \\
\text { per Nucleon }\end{array}$ & 280.037 & 725.4786 & 301.5832 & $\mathrm{MeV}$ \\
\hline $\begin{array}{c}B \rho \\
\text { B } \rho\end{array}$ & 5.1674829 & 12.7925945 & 5.3894886 & $\mathrm{Tm}$ \\
\hline $\begin{array}{c}\text { Bagnetic } \rho / \rho \\
\text { Field Setpoint }\end{array}$ & 3726.84 & 9226.14 & 3886.95 & Gauss \\
\hline $\begin{array}{c}\text { Calculated } \\
\text { MM Current }\end{array}$ & 1534 & 3797 & 1600 & Amps \\
\hline $\begin{array}{c}\text { MM Current } \\
\text { Setpoint }\end{array}$ & 1563 & 3878 & 1629 & Amps \\
\hline
\end{tabular}


Table 7: Iron Parameters at Extraction

\begin{tabular}{|c|c|c|c|c|}
\hline Parameter & $\mathrm{Fe}^{20+}$ & $\mathrm{Fe}^{20+}$ & $\mathrm{Fe}^{20+}$ & Unit \\
\hline$m c^{2}$ & 52.0928437 & 52.0928437 & 52.0928437 & $\mathrm{GeV}$ \\
\hline Date & 30 May 03 & 18 June 03 & 19 June 03 & \\
\hline$h f$ & $3.68724(60)$ & $3.89245(60)$ & $3.90872(60)$ & $\mathrm{MHz}$ \\
\hline$h$ & 3 & 3 & 3 & \\
\hline$T=1 / f$ & 0.81362 & 0.77072 & 0.76751 & $\mu \mathrm{s}$ \\
\hline $\begin{array}{c}\text { Kinetic E } \\
\text { per Nucleon }\end{array}$ & 725.4786 & 979.1155 & 1005.3420 & $\mathrm{MeV}$ \\
\hline $\begin{array}{c}B \rho \\
\text { B } \rho\end{array}$ & 12.7925945 & 15.5733095 & 15.85321093 & $\mathrm{Tm}$ \\
\hline $\begin{array}{c}\text { Magnetic } \rho \\
\text { Field Setpoint }\end{array}$ & 9226.14 & 11231.62 & 11433.48 & Gauss \\
\hline $\begin{array}{c}\text { Calculated } \\
\text { MM Current }\end{array}$ & 3797 & 4622 & 4705 & Amps \\
\hline $\begin{array}{c}\text { MM Current } \\
\text { Setpoint }\end{array}$ & 3878 & 4822 & 4934 & Amps \\
\hline
\end{tabular}


Table 8: Carbon, Iron, and Titanium Parameters at Extraction

\begin{tabular}{|c|c|c|c|c|}
\hline Parameter & $\mathrm{C}^{6+}$ & $\mathrm{Fe}^{20+}$ & $\mathrm{Ti}^{18+}$ & Unit \\
\hline$m c^{2}$ & 11.1748622 & 52.0928437 & 44.6540277 & $\mathrm{GeV}$ \\
\hline Date & 9 June 03 & 19 June 03 & 23 June 03 & \\
\hline$h f$ & $2.9208(6)$ & $3.90872(60)$ & $3.91144(60)$ & $\mathrm{MHz}$ \\
\hline$h$ & 3 & 3 & 3 & \\
\hline$T=1 / f$ & 1.02712 & 0.76751 & 0.76698 & $\mu \mathrm{s}$ \\
\hline $\begin{array}{c}\text { Kinetic E } \\
\text { per Nucleon }\end{array}$ & 301.5832 & 1005.3420 & 1009.9121 & $\mathrm{MeV}$ \\
\hline$B \rho$ & 5.3894886 & 15.85321093 & 15.14496712 & $\mathrm{Tm}$ \\
\hline$B \rho / \rho$ & 3886.95 & 11433.48 & 10922.69 & Gauss \\
\hline $\begin{array}{c}\text { Magnetic } \\
\text { Field Setpoint }\end{array}$ & 3962 & 11600 & 11100 & Gauss \\
\hline $\begin{array}{l}\text { Calculated } \\
\text { MM Current }\end{array}$ & 1600 & 4705 & 4495 & Amps \\
\hline $\begin{array}{l}\text { MM Current } \\
\text { Setpoint }\end{array}$ & 1692 & 4934 & 4663 & Amps \\
\hline
\end{tabular}




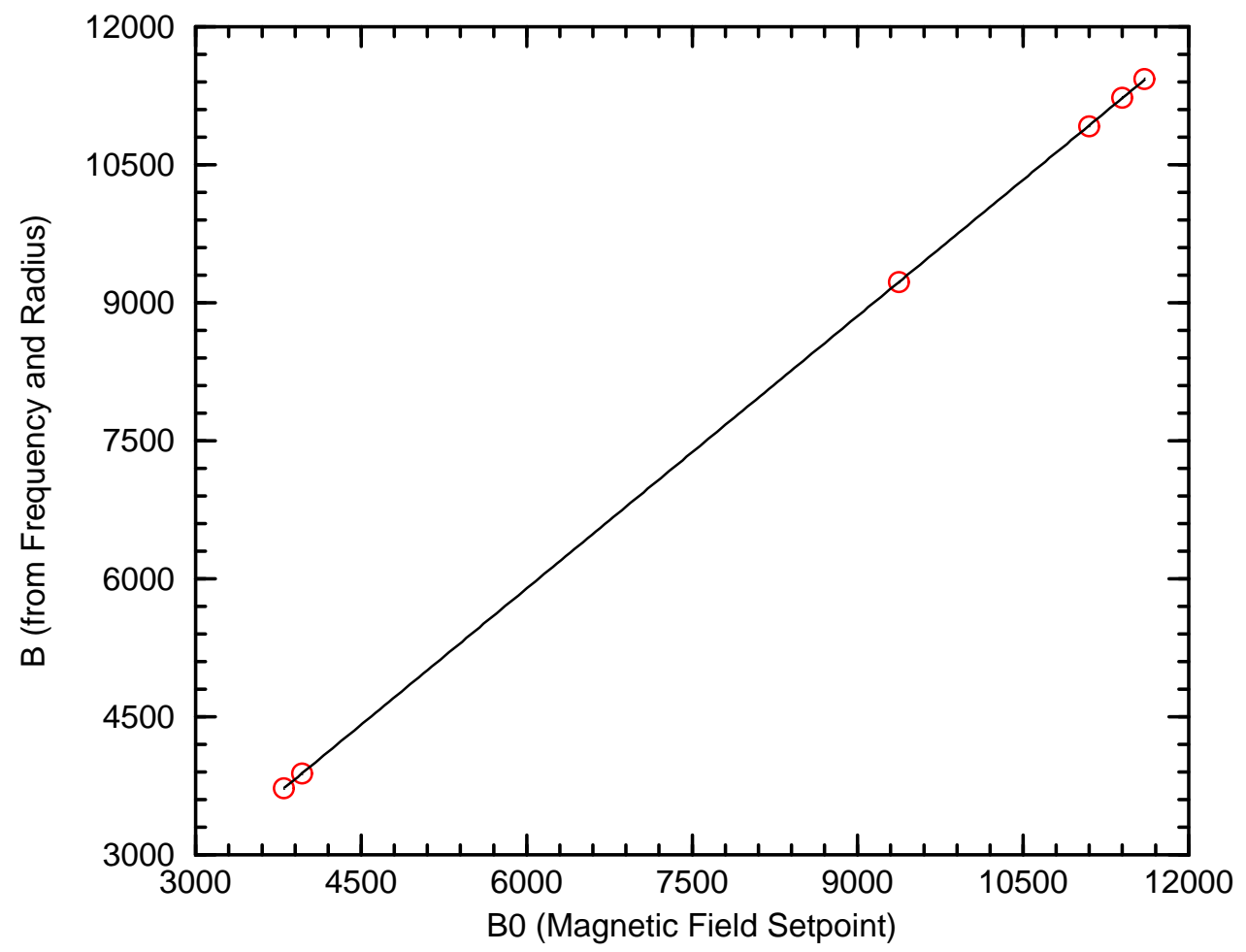

Figure 7: Plot of $B$ and $B_{0}$ data from Table 9 with fitted line. Units are Gauss. $B$ is the field at extraction determined from frequency and radius. $B_{0}$ is field setpoint in the Booster Main Magnet program. The line $B=m B_{0}+b$ is fitted to the data points. This gives fitted parameters $m=0.98710(81)$ and $b=-24.9(7.4)$ Gauss. The indicated errors were obtained assuming a statistical error of $\sigma=6.7$ Gauss for each value of $B$. This gives a $\chi^{2}$ per dof of 1 . 


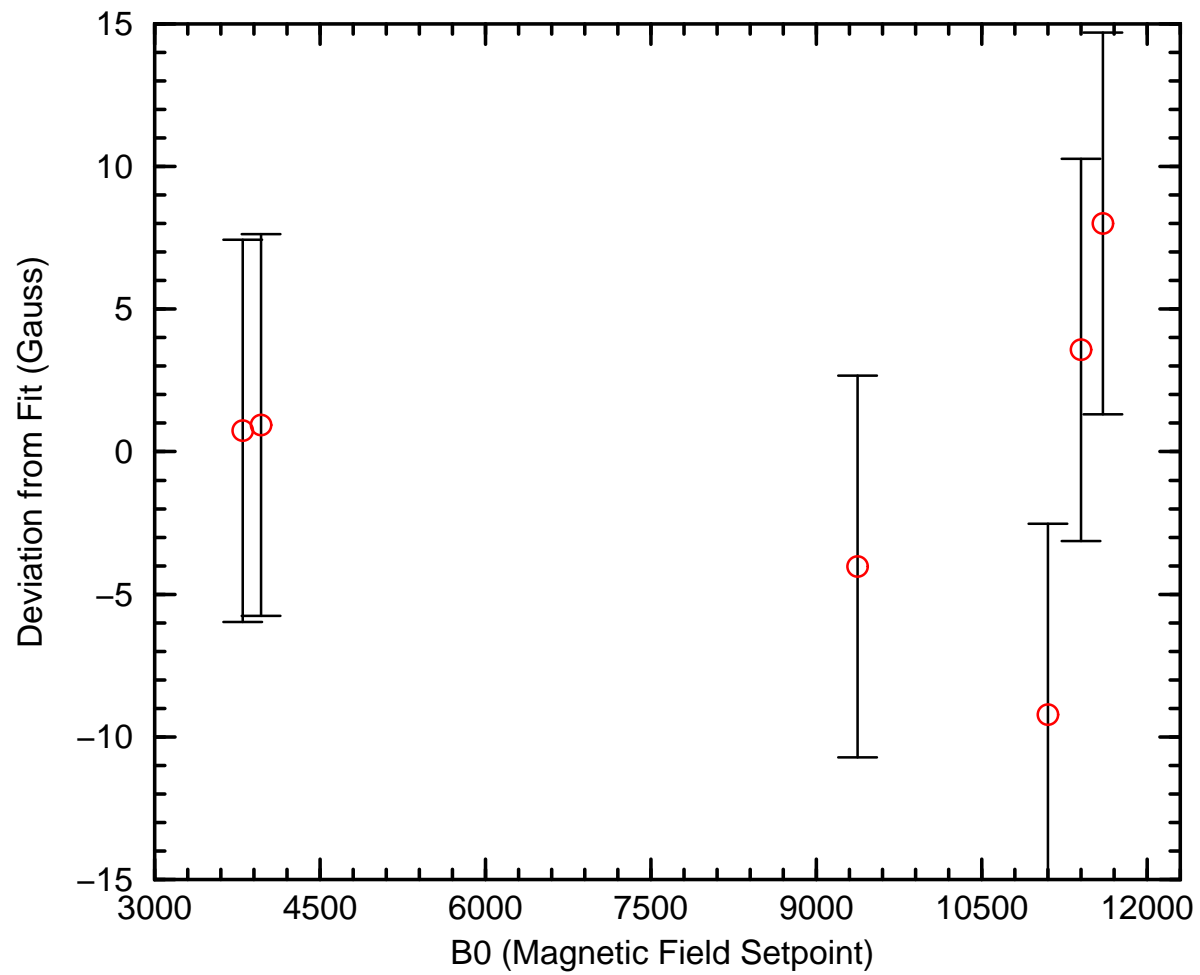

Figure 8: Deviation of Figure 7 data points from fitted line. Units are Gauss. The indicated errors are \pm 6.7 Gauss. 
Table 9: Summary of Rigidities and Fields at Extraction. Units are Tm and Gauss. $B \rho$ and $B$ are determined from frequency measurements and radius. $B_{0}$ is the field setpoint in the Booster Main Magnet program.

\begin{tabular}{|c|c|c|c|}
\hline Date & $B \rho$ & $B$ & $B_{0}$ \\
\hline 27 May 03 & 5.1674829 & 3726.84 & 3800 \\
\hline 30 May 03 & 12.7925945 & 9226.14 & 9376 \\
\hline 9 Jun 03 & 5.3894886 & 3886.95 & 3962 \\
\hline 18 Jun 03 & 15.5733095 & 11231.62 & 11400 \\
\hline 19 Jun 03 & 15.85321093 & 11433.48 & 11600 \\
\hline 23 Jun 03 & 15.14496712 & 10922.69 & 11100 \\
\hline
\end{tabular}

